J3eA, Journal sur l'enseignement des sciences et technologies de l'information et des systèmes,

Volume 3, Hors-Série 1, 24 (2004)

DOI : http://dx.doi.org/10.1051/bib-j3ea:2004624

(C) EDP Sciences, 2004

\title{
Clavier virtuel multimodal : applications à des situations de handicap
}

\author{
J.P. Mazeau ${ }^{1}$, J. Lopez Krahe ${ }^{1}$ et C. Cesarano ${ }^{2}$ \\ ${ }^{1}$ Université Paris VIII \\ Laboratoire LTCM, GRAII - UFR 6 \\ 2 rue de la liberté \\ F-93526 Saint-Denis CEDEX 02, France \\ ${ }^{2}$ CNEFEI \\ 58-60 avenue des Landes \\ F-92160 Suresnes, France
}

mazeau@univ-paris8.fr, jlk@ai.univ-paris8.fr, cesarano@free.fr 


\title{
Clavier virtuel multimodal : Applications à des situations de handicap
}

\author{
Jean-Paul Mazeau ${ }^{1}$, Jaime Lopez Krahe ${ }^{1}$, Serge Cesarano ${ }^{2}$ \\ ${ }^{1}$ Université Paris VIII - Laboratoire LTCM, GRAII - UFR.6 \\ 2 rue de la liberté - Saint - Denis 93.526 Cedex 02 \\ ${ }^{2}$ CNEFEI, 58 - 60 Av des Landes 92160 Suresnes \\ mazeau@univ-paris8.fr,jlk@ai.univ-paris8.fr, cesarano@free.fr
}

\begin{abstract}
Résumé - Cette communication se situe dans le thème « interfaces homme-homme et homme-machine » et dans la catégorie des interfaces à manipulations directes. Elle propose de communiquer avec une machine au moyen d'un clavier virtuel implanté dans un volume pouvant atteindre plusieurs dizaines de mètres cubes. Dans ce volume nous "installons" des touches virtuelles qui sont définies par des régions fixes ou mobiles. Comme les touches traditionnelles, ces touches interactives vont permettre d'établir une communication gestuelle directe entre les personnages qui évoluent dans le volume interactif, et les ordinateurs auxquels elles sont « reliées ».

Nous présentons ici trois applications dans le contexte des personnes handicapées, une première concerne des jeunes autistes, une seconde un clavier virtuel pour handicapes moteurs, et la dernière une aide à la rééducation fonctionnelle. Nous tenterons de faire apparaître :

- les spécificités propres à ce type de "sans clavier" qui est utilisé corporellement, de l'extérieur ou de l'intérieur,

- les stratégies de déclenchement qui donnent lieu à des modes d'expression corporelle spécifiques,

- des exemples de composition de clavier, la nature des informations délivrées, les applications auxquelles ils sont destinés.
\end{abstract}

Mots clés : Interface corporelle, désignation gestuelle, ordinateur sans clavier, représentation visuelle, multimodalité, clavier adapté.

\section{LE CLAVIER VIRTUEL}

Le clavier virtuel a les caractéristiques de l'écran tactile, mais il peut s'inscrire dans un espace variable non délimité par la surface d'un écran. Dans le cas le plus général, nous admettons qu'un clavier virtuel est inscrit dans un espace 3D occupé par un ensemble de volumes qui permettent de communiquer avec des ordinateurs. Ces volumes se substituent aux touches matérielles des claviers ou virtuelles des écrans tactiles.

\section{Figure 1}

\section{A) Le clavier}

La Fig. 1 nous montre un clavier virtuel de 4 touches dans lequel évoluent les utilisateurs. L'espace clavier est délimité par les traits en pointillés. Dans cet exemple, le clavier s'inscrit dans un volume de plusieurs dizaines de mètres cubes. Ce volume est délimité par un plancher réel sur lequel se déplacent les utilisateurs, par un plafond virtuel qui limite vers le haut la partie active de l'espace et par quatre murs virtuels.

Dans cet exemple nous montrons trois utilisateurs. Le premier se trouve à l'intérieur du clavier, les deux autres à l'extérieur. Ce clavier est indifféremment utilisable à sa périphérie ou dans sa "masse". Le clavier étant un espace vide, le passage de l'intérieur à l'extérieur n'est pas contrarié par un obstacle physique.

L'espace clavier contient les utilisateurs ainsi que des objets réels spécifiques aux divers environnements: des meubles, des décors, des obstacles...

\section{B) Les touches}

Chaque touche est un volume invisible caractérisé par sa section, sa hauteur et sa position. Ces touches virtuelles sont modifiables en cours d'utilisation du clavier. C'est ainsi qu'une touche peut apparaître, disparaître, changer d'emplacement ou de forme. Chacune de ces évolutions provoque une modification dans l'interaction avec le clavier. Ici le clavier est utilisé directement par le corps. C'est l'implantation des touches et les consignes qui vont imposer un type d'activité corporelle aux utilisateurs.

La Fig.1 fait apparaître deux types de touches: celles qui traversent l'espace du sol au plafond, celles qui "flottent" dans l'espace. Remarquons que la touche flottante est positionnée par rapport à des axes, qui en indiquent les limites de déplacement.

Les touches mobiles. Nous distinguerons deux catégories de touches mobiles, les touches :

- manipulées par les utilisateurs quand à leur forme et leur implantation.

- manipulées par le système de contrôle; parmi ces dernières, nous avons développé un système qui permet à une touche de suivre un utilisateur ou un objet mobile identifié.

Cette approche est utilisée dans des applications de jeux, d'apprentissage de parcours, de rééducation fonctionnelle, d'entrainement sportif...

\section{C) Les utilisateurs}

Les utilisateurs ou les objets réels qui occupent le clavier peuvent être considérés comme des touches réelles mobiles ou fixes. Le problème consiste alors à étudier les collisions entre les touches réelles et les touches virtuelles représentées par les volumes. 


\section{D) La représentation}

\section{Figure 2}

Pour que le clavier soit utilisable "consciemment" il est indispensable que l'utilisateur puisse se repérer. Le clavier est donc un ensemble constitué de l'espace interactif, mais aussi d'éléments capables de représenter l'espace, les utilisateurs qui y évoluent et les relations géographiques entre ces utilisateurs et les touches. Ces relations ne sont pas nécessairement du type tout ou rien. Nous verrons que les touches peuvent délivrer des informations complexes permettant de commander des objets dans des espaces 2D ou $3 \mathrm{D}$, des volumes sonores etc...

Ces représentations sont supportées par des images, qui contiennent les touches, les utilisateurs et les éventuels objets réels ou virtuels qui occupent les espaces.

Remarque: certains danseurs utilisent la représentation pendant les phases de répétition. Quand le trajet est parfaitement acquis ils évoluent sans repérage, comme une dactylo utilise le clavier alphanumérique les yeux fermés.

\section{E) Les relations}

Une relation doit s'établir entre les utilisateurs et les volumes représentant les touches. Si l'utilisateur pénètre dans ces volumes, directement ou au moyen d'objets qu'il manipule, il va déclencher le processus interactif. Pour que la relation s'établisse il est nécessaire que le corps ou les parties du corps qui interagissent soient identifiables. Prenons quelques exemples d'éléments susceptibles d'activer les touches: la couleur de la peau, la forme du coude, un objet jaune; Nous admettons que le déclenchement d'une même touche par l'objet jaune ou par la couleur de la peau provoque des interactions différentes, comme si l'action provoquée par le pouce sur un clavier était distincte de celle provoquée par l'index.

\section{F) Les conditions de déclenchement}

Voici quelques conditions de déclenchement :

- Un objet interactif pénètre pendant plus de 2 secondes dans une touche,

- Le seuil de contact est supérieur à une certaine grandeur.

En général, nous considérons que si la manière de pénétrer dans les touches correspond à un certain modèle alors nous activons la communication entre le clavier et l'ordinateur.

\section{G) La navigation}

Contrairement à un clavier actionné par un contact physique, le clavier virtuel est contrôlé par un "contact virtuel". Se pose alors le problème de la définition du contact virtuel.

Si nous reprenons l'exemple du déclencheur "chaussure noire", nous considérons que chaque passage de la chaussure noire dans un volume va provoquer une interaction touche-ordinateur. Donc toute "traversée" de touche qui se trouve sur un itinéraire va provoquer une interaction, ce qui correspond au déclenchement des touches "survolées" d'un clavier mécanique. D'où la nécessité d'éviter les touches non pertinentes ou de neutraliser temporairement, lors des trajets les signaux déclencheurs manipulés par l'utilisateur.

\section{Figure 3a Figure 3b Figure 3c}

Les Fig. 3a, 3b représentent deux configurations où toutes les touches sont accessibles directement, sans risque de collision. Dans la Fig.3a, l'utilisateur est "enfermé" à l'intérieur de son clavier. Dans la Fig. 3b les touches ne sont pas toutes accessibles directement. Il est indispensable de naviguer pour éviter les déclenchements intempestifs, ou de "neutraliser" les parties actives (signaux) lors du passage dans les touches à ne pas déclencher.

La Fig.3c nous montre un exemple de parcours imposé par un trait directeur. Si nous utilisons ce clavier pour commander un synthétiseur, nous "attachons" un son à chaque touche. L'utilisateur qui suit le trait directeur joue la mélodie. La mélodie est écrite dans le dessin qui représente le clavier. Des paramètres tels que l'attaque ou le volume peuvent être contrôlés. En effet, c'est l'utilisateur seul qui décide de sa vitesse de déplacement (contrôle du rythme), ainsi que de l'accélération qu'il imprime à ses gestes (contrôle de l'attaque ou du niveau).

Cet exemple montre que l'interprète musicien est aussi un danseur puisque c'est par l'expression corporelle qu'il va directement interpréter la musique.

\section{H) La simultanéité}

Elle consiste à traiter simultanément l'ensemble des touches activées à un instant donné. Dans le dessin de la Fig.1, trois personnes sont représentées. Nous supposons que le clavier peut communiquer l'ensemble des informations concernant tous les utilisateurs. Le clavier doit avoir les mêmes fonctionnalités qu'un piano capable de déclencher en même temps un ensemble de notes, et d'en contrôler les divers paramètres. En règle générale, il s'agit de traiter simultanément et en temps réel, toutes les touches actives du clavier.

Le temps réel est défini ici par rapport à la durée d'une trame vidéo (20ms). L'ensemble des informations doit être mis à jour à chaque trame.

\section{QUELQUES EXEMPLES DE TOUCHES ET DE CLAVIERS}

\section{A) Touche tout ou rien}

\section{Figure 4}

Cette touche est validée quand la surface entre la main et la touche est supérieure au seuil. 
B) Touche analogique

\section{Figure 5}

L'information transmise est la surface entre la main et la touche. Aux deux positions de la main vont correspondre deux valeurs différentes.

\section{C) Clavier de 5 touches analogiques}

Ce clavier permet le contrôle simultané d'une direction (main droite) d'une vitesse (main gauche), d'une hauteur (pied droit).

\section{Figure 6}

Les touches T2 et T3 contrôlent la hauteur de l'objet piloté. L'information transmise à l'ordinateur est fonction du rapport des surfaces $\left(\mathrm{T} 3{ }^{*}\right.$ pied), $(\mathrm{T} 2 *$ pied). Le contrôle de la direction (touches T4,T5) est réalisé sur le même principe. La touche $\mathrm{T} 1$ est une touche analogique du type (2). Les formes des touches, ainsi que les emplacements respectifs peuvent être ajustés par les utilisateurs. Ainsi, l'utilisateur peut disposer d'un fichier de configuration du clavier qui lui est personnel.

\section{D) Repérage simultané de deux mains}

Dans cet exemple, nous avons un clavier de 60 touches parallélépipédiques séparées par un couloir.

Nous avons affaire à un découpage matriciel permettant la localisation des deux mains dans deux repères de $5 * 12$ touches. Les touches actives sont représentées par les zones noircies.

\section{E) Une méthode permettant à l'utilisateur d'implanter les touches}

Nous proposons un type de touche qui permet à l'utilisateur d'installer gestuellement des touches de commande dans un espace clavier. Il peut ainsi les implanter en fonction de son application et de ses choix ou contraintes.

Il s'agit ici de faire poursuivre un objet réel par un objet graphique. Cette opération se décompose en trois phases:

\section{Désignation, déplacement, fin de l'installation.}

Quand l'objet déclencheur, ici la main, pénètre dans une des touches, la touche poursuite prend la direction qui lui est associée.

Cette opération se termine si la main est retirée par un geste rapide. La touche poursuite est remplacée par la touche clavier. Cette opération peut être répétée jusqu'à l'installation complète du clavier.

\section{F) Le clavier réparti}

Par définition, un clavier réparti se situe dans des espaces géographiques distincts. Il permet à plusieurs personnes éloignées de communiquer, bien que le clavier soit représenté dans un environnement unique. Cela signifie que les utilisateurs distants sont rassemblés dans le même espace de représentation. Nous pouvons parler de " télé clavier".

\section{CONTEXTE HANDICAP}

\section{A) Claviers virtuels pour handicapés moteurs}

Définition du projet: Réaliser un clavier virtuel adaptable aux possibilités d'utilisateurs présentant un déficit moteur aux membres supérieurs. Il s'agit d'un problème important de communication et d'accessibilité à l'ordinateur. Les solutions actuelles impliquent la construction d'un clavier adapté à chaque cas (contacteurs pneumatiques, clavier physique spécifique, accès par arborescences, etc.). Le projet apporte une solution générale et adaptable a chaque cas par logiciel.

Objectifs du projet : Le clavier virtuel obtenu peut être mémorisé pour un individu donné, mais aussi très facilement redéfini en fonction des possibilités motrices variables d'un jour à l'autre, de l'évolution (réadaptation ou dégradation) de l'handicap.

Public concerné : Personnes lourdement handicapées, présentant des possibilités motrices réduites des membres supérieurs :

* myopathes ayant une mobilité réduite et très peu de puissance musculaire

* infirmes moteurs cérébraux présentant une atteinte des membres supérieurs avec un champ d'action réduit

* syndromes spastiques avec de grandes contractions musculaires où un filtrage est possible par logiciel

* syndromes athétosiques où une moyenne des déplacements permet d'estomper le côté erratique des mouvements

Adaptations possibles: La forme, la taille, et le nombre de touches du clavier sont redéfinis à tout instant par une séquence rapide d'étalonnage au moyen d'un jeu.

La forme du clavier, situé dans l'espace d'évolution du membre actif, ne sera pas forcément rectangulaire, mais pourra s'adapter aux mouvements possibles.

Le nombre des touches n'est pas fixe, il peut varier de 1 à 30 environ. Un système permettant d'affecter plusieurs caractères à une même touche est prévu. Un retour du caractère sélectionné est présent sur l'affichage.

Le mode de balayage du clavier est aussi paramétrable, par dichotomie ou balayage par quadrant, case à case ou lignes/colonnes.

Le clavier est défini après une phase d'auto adaptation en mode jeu étalonnage.

Dans un premier temps, on étudie le système de validation des touches les plus adéquats à la personne. Ceci peut être une collision spécifique, un système pneumatique, un connecteur, etc.

Par la suite un jeu de poursuite permet de définir la forme et l'espace utile du clavier. Des mesures sur l'exactitude des collisions avec la cible donnent les informations sur la précision des mouvements utiles, et permet de définir les zones de meilleure résolution. 
Il s'agit d'un «apprentissage » de l'individu par la machine, avec l'objectif de proposer un clavier adapté a chaque situation.

Par la suite on propose les arborescences d'accès, qui peuvent être modifiées en fonction de l'ergonomie de l'utilisateur, et le clavier peut être mémorisé.

\section{B) Autisme et clavier virtuel}

Les difficultés de communication, d'interaction sociale, l'absence de contact visuel, la carence d'expressions faciales et de gestes, sont quelques caractéristiques des personnes autistes.

Nous avons tenté une expérience de manière à évaluer les capacités des jeunes autistes à interagir avec l'environnement et à s'approprier de l'espace d'interaction au moyen des sons et des images.

Cette expérience à été menée par des éducateurs qui ont pu disposer librement d'un prototype installé dans leur centre entre mai et juin 2003.

Une évaluation sur les interactions diverses (corporelles, visuelles, auditives), l'émotion et l'expression ainsi que sur la capacité d'apprentissage, est en cours de dépouillement. [Les données chiffrées seront présentées dans la version définitive de la communication.]

En fonction des premiers résultats (capacité d'interaction et d'apprentissage pour quelques jeunes), d'autres perspectives s'ouvrent pour l'utilisation positive de cet équipement avec un objectif de communication et collaboration chez les personnes autistes.

Objectifs du projet: Mettre sur place des jeux collaboratifs qui impliquent une collaboration de deux ou plusieurs personnes pour obtenir un objectif commun...

\section{Exercice 1:}

Cacher $\mathrm{n}$ taches mobiles en même temps, ceci implique la collaboration de deux $n / 2$ joueurs avec deux mains ou $n$ joueurs avec une main. Le score avance en renforcement de la collaboration.

\section{Exercice 2 :}

Cacher à plusieurs une grande tache avec les corps à plusieurs pour obtenir le gain. Cette tache peut changer de forme et se déplacer dans une deuxième phase du jeu.

\section{C) Réadaptation fonctionnelle:}

Voici un projet en cours de réalisation: Aide et évaluation des rééducations.

Définition du projet : Observer, guider et évaluer des rééducations

Objectifs du projet: Rendre plus motivante la rééducation (jeux programmés)

Permettre la programmation d'exercices s'adaptant aux progrès du patient.

Evaluer les progrès au fil des séances

Entraîner à des mouvements spécifiques
Public concerné : Personnes présentant un handicap moteur permanent ou passager et en cours de rééducation.

\section{Exercice 1:}

Programmer des mouvements en repérant les amplitudes extrêmes par des objets virtuels sonores. La position de ces objets peut ainsi varier dans le temps, permettant une adaptation des exercices. La caméra pourra être placée à l'horizontale pour repérer des déplacements des membres supérieurs. Les positions significatives peuvent être mémorisées pour garder l'historique des exercices.

\section{Exercice 2:}

Evaluer les temps de réalisation et la fréquence d'exercices proposés, permettant ainsi de suivre l'adaptation à l'effort de l'individu. Les exercices pourront être exécutés en fonction de stimuli sonores.

\section{Exercice 3 :}

Entraînement à un geste technique lié à un objet virtuel : balle de tennis, de ping-pong, ballon ..., exercices avec une composante ludique.

\section{CONCLUSION}

Lors des expérimentations, nous avons pu définir des limites à l'interaction gestuelle directe avec des capteurs de type caméra, limites dues en particulier aux fourchettes d'éclairage, à l'emplacement des caméras, à la difficulté de préciser les objets interactifs mais jamais à la vitesse de déplacement des utilisateurs.

Ce clavier virtuel doit permettre des applications nombreuses dans des situations de handicap, aussi bien pour palier cette situation en tant qu'aide technique adaptable par logiciel que comme outil de rééducation ou de réadaptation fonctionnelle. La présentation du matériel dans le cadre de la journée, doit permettre à des utilisateurs potentiels de trouver d'autres applications.

\section{BIBLIOGRAPHIE}

- Mazeau JP, Architecture de traitement d'images interagissantes en temps réel. Brevet d'invention $\mathrm{N}^{\circ}$ 02/16189, Paris 19/12/2002.

- Martin JC, Interfaces multimodales, Thèse, Paris 1995

- J-P Mazeau, «Un espace interactif polyvalent pour une communication corporelle directe. », PRC Communication H/M (CNRS/Ministère de la recherche) 6ème Journées du GT Réalité Virtuelle, 13/03/99, CNET Issy les Moulineaux - J-P Mazeau, "Jongler avec des objets virtuels ", 6ème convention des metteurs en scènes, 26/01/97, Paris. 
- J-P Mazeau, « Des instruments commandés gestuellement », Forum IRCAM, 14/11/97, Paris.

- J-P Mazeau, X Bryche, « Clavier virtuel tridimensionnel de grande dimension », ERGO.IA 96, 9/10/96, Biarritz. 
6. FIGURES
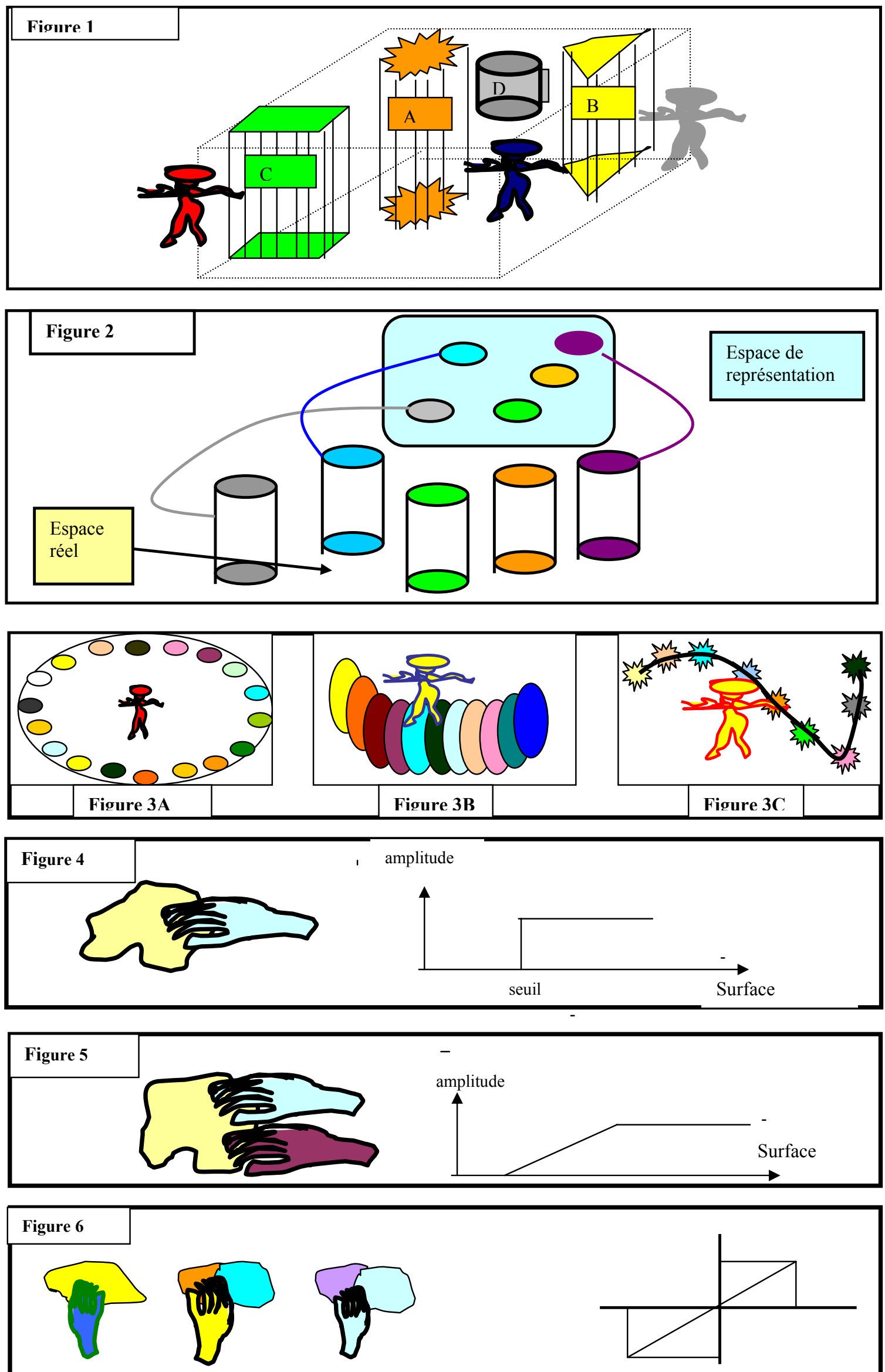